\title{
Polymorphisms of Genes Encoding Multidrug Resistance Proteins as a Predictive Factor for Second-Line Docetaxel Therapy in Advanced Non-small Cell Lung Cancer
}

\author{
Michał Szczyrek $^{1,2} \cdot$ Radoslaw Mlak $^{3} \cdot$ Pawel Krawczyk $^{1} \cdot$ Kamila Wojas-Krawczyk $^{1}$. \\ Tomasz Powrózek ${ }^{1}$ • Aneta Szudy-Szczyrek ${ }^{4}$ - Agnieszka Zwolak ${ }^{2}$. Jadwiga Daniluk ${ }^{2}$ • \\ Janusz Milanowski ${ }^{1}$
}

Received: 2 October 2015 / Accepted: 8 December 2016/Published online: 17 December 2016

(C) The Author(s) 2016. This article is published with open access at Springerlink.com

\begin{abstract}
Multidrug resistance (MDR) remains a substantial problem in chemotherapy. The purpose of the study was to investigate potential factors, including $M D R$ genes polymorphisms, that could be used in qualification for second-line docetaxel therapy in non-small cell lung cancer (NSCLC) patients after failure of platinum based chemotherapy. Study group comprised of 58 Caucasian subjects. Evaluation of Single Nucleotide Polymorphisms (SNPs) of $A B C C 2 / M R P 2$ and $A B C B 1 / M D R 1$ genes was performed using the High Resolution Melting (HRM) technique. TUBB3 gene expression was evaluated on RNA isolated from tumor tissue. Results with $p$ value of $<0.05$ were considered significant. Factors associated with reduced risk of disease progression included good performance status (PS), long period between diagnosis and docetaxel treatment, and smoking for $<10$ packyears. Disease control occurred more often in patients with $\mathrm{G} / \mathrm{G}$ genotype of the $A B C C 2 / M R P 2$ gene. Median overall survival was 4.25 months. Factors such as: good PS, disease control after docetaxel, long period from diagnosis to docetaxel, lack of significant weight loss, and third-line treatment were associated with prolongation of patients survival. Overall survival probability was significantly lower in patients
\end{abstract}

Michał Szczyrek

mszczyrek@yahoo.co.uk

1 Department of Pneumonology, Oncology and Allergology, Medical University of Lublin, Lublin, Poland

2 Chair of Internal Medicine and Department of Internal Medicine in Nursing, Medical University of Lublin, Lublin, Poland

3 Department of Human Physiology, Medical University of Lublin, Lublin, Poland

4 Chair and Department of Haematooncology and Bone Marrow Transplantation, Medical University of Lublin, Lublin, Poland with significant weight loss, poor PS, lack of disease control after docetaxel, and without third-line treatment. Factors that characterized the highest risk of survival shortening were: inability to apply third-line treatment, lack of best response to first-line therapy, poor PS, and $\mathrm{C} / \mathrm{G}$ or $\mathrm{G} / \mathrm{G}$ genotypes of $A B C C 2 / M R P 2$ gene. We concluded that assessed factors had mainly prognostic and not predictive value. Finding reliable molecular predictors for second line docetaxel therapy requires further clinical trials.

Keywords NSCLC $\cdot$ Docetaxel $\cdot T U B B 3 \cdot A B C C 2 \cdot M D R 1$

\section{Introduction}

Non-small cell lung cancer (NSCLC) accounts for $80 \%$ of lung neoplasms. Early stage of the disease is an indication for surgery, while locally advanced or metastatic NSCLC is treated with chemotherapy, radiotherapy and molecularly targeted therapies. Response to first-line therapy is observed in 30-40\% of patients, second line of treatment is even less effective.

Docetaxel is a well-known antimitotic drug that can be used in second-line treatment after failure of prior platinum based chemotherapy. It reversibly binds to microtubules ( $\beta$ tubulins), stabilizes them and prevents their depolymerization. It also leads to phosphorylation of BCL-2, which is an apoptosis-blocking oncoprotein. Therefore, it works by interfering with cell division and inducing cell apoptosis. Docetaxel is effective in about $10 \%$ of patients.

The multidrug resistance (MDR) remains a substantial problem in development of resistance to chemotherapeutic treatment. The overexpression of ATP-binding cassette $(\mathrm{ABC})$ transporters is responsible for cell removal of several classes of chemotherapeutics including taxanes, vinca 
alkaloids and nucleoside analogs, as well as physiologic substrates including leukotrienes and glutathione. The $\mathrm{C}$ subfamily of $\mathrm{ABC}$ proteins is alternatively known as the $\mathrm{ABCC}$ proteins or the multidrug resistance protein (MRP) subfamily. Pglycoprotein, a pivotal member of $\mathrm{ABC}$ transporters, is the product of multidrug resistance gene $(A B C B 1 / M D R 1)$. Polymorphisms (including single nucleotide polymorphisms, SNPs) of $M D R$ genes are responsible for differences in expression and activity of several proteins involved in the removal of cytostatics form tumor cells.

The purpose of this study was to investigate potential clinical and molecular factors, including $M D R$ genes polymorphisms, that could be used in qualification for second-line docetaxel therapy in patients with NSCLC after failure of first-line therapy.

\section{Material and Methods}

The study group comprised of 58 Caucasian patients with locally advanced or metastatic NSCLC after failure of first-line chemotherapy. The staging of disease was determined according to the TNM classification (VII edition), and response to treatment was evaluated according to RECIST version 1.1. Performance status of patients was assessed in ECOG-WHO scale. All patients received first-line treatment with platinumbased chemotherapy. In all patients docetaxel at a dose of $75 \mathrm{mg} / \mathrm{m}^{2}$ was used as a second line treatment. Detailed characteristics of the patients are presented in Table 1.

$T U B B 3$ gene expression was evaluated on RNA isolated from formalin fixed paraffin embedded tumor tissue. Isolation of RNA was carried out using the RNeasy FFPE Kit (Qiagen, Canada). SNPs of $A B C C 2 / M R P 2$ (rs12762549, g.99861014C > G) and $A B C B 1 / M D R 1$ (rs1045642, c.3435 T > C) genes were studied on DNA isolated from peripheral blood leucocytes, using the QIAmp Blood Mini Kit (Qiagen, Canada). Evaluation of the quality and quantity of extracted DNA/RNA was carried out using the BioPhotometer plus (Eppendorf, Germany).

TUBB3 gene expression was assessed using Real-Time Quantitative Reverse Transcription PCR. Reverse transcription reaction was carried out using High Capacity cDNA Reverse Transcription Kit (Life Technologies, USA) with specific primers and reverse transcriptase. Expression of mRNA for TUBB3 gene was analyzed on ABI PRISM 7500 equipment (Life Technologies, USA) using TaqMan Gene Expression Assay (Life Technologies, USA).

The evaluation of SNPs of $A B C C 2 / M R P 2$ and $A B C B 1 /$ MDRl genes was performed using the PCR HRM (High Resolution Melting) technique. Reaction parameters for PCR and HRM were based on the manufacturer's instructions from the KAPA FAST HRM PCR set (Kapa Biosystems, USA). PCR HRM was carried out on Eco Illumina (Illumina, USA) real-time PCR equipment.
Statistical analysis of data was performed using the MedCalc 10 (MedCalc Software, Belgium) and Statistica 10 (Statsoft, USA) software. Hardy-Weinberg equilibrium and the impact of demographic, clinical and genetic factors on the response to treatment and 6-month survival of the patients was estimated with the use of the Chi Square $\left(\chi^{2}\right)$ test. Cox regression model with a stepwise selection of clinical and molecular factors with the minimum AIC was used to determine the model having the greatest impact on overall survival. Kaplan-Meier estimation method was used to compare the probability of overall survival in patients with different clinical, demographic and genetic characteristics. Large cell carcinoma and NOS (not-otherwise specified) NSCLC were excluded from statistical analysis due to its confusing nature and small number of cases. Also smoking status was excluded due to the fact, that. Most of patients $(94.8 \%)$ were classified as heavy smokers. In all the performed test, results with $p$ value of $<0.05$ were considered as statistically significant.

\section{Results}

In the distribution of genotypes of the $A B C C 2$ and $M D R 1$ genes there were no deviations from the Hardy-Weinberg equilibrium. CC genotype of $A B C C 2$ gene was present in $21.1 \%, \mathrm{CG}$ in $38.5 \%$ and $\mathrm{GG}$ in $40.4 \%$ of patients. In the case of the MDR1 gene, genotypes CC, CT and TT were found in $15.4 \%, 44.2 \%$ and $40.4 \%$ of subjects. The distribution of genotypes of $A B C B 1 / M D R 1$ and $A B C C 2 / M R P 2$ genes did not depended on demographic and clinical factors.

The median value of $\triangle \mathrm{Ct}$ for TUBB3 gene (relative to the reference gene $A C T B-\beta$-actin) was 5.9 cycles ( \pm 5.41 cycles). Low expression of mRNA (below median value of $\triangle \mathrm{Ct}$ ) for the TUBB3 gene have been reported in $54.6 \%$, while high expression in $45.4 \%$ of patients. Expression of mRNA for the TUBB3 gene didn't depended on demographic or clinical factors.

Among the studied patients, there were no complete remissions. Disease control was achieved in 14 patients (24.1\%), 44 patients $(75.9 \%)$ experienced early progression. Partial remission was observed in 4 patients $(6.9 \%)$ In patients who reached disease control, median time to progression after docetaxel was 5 months ( \pm 10.5 months).

Factors associated with reduced risk of early progression included: good PS (HR $=0.57,95 \% \mathrm{CI}: 0.38-0.85$, $p=0.0019)$, long (>12 months) period between diagnosis of NSCLC and docetaxel treatment (HR $=0.69,95 \%$ CI: 0.47 $0.99, p=0.0437)$. Disease control occurred significantly more often (HR $=0.71,95 \% \mathrm{CI}: 0.52-0.98, p=0.0323$ ) in patients with GG genotype of the $A B C C 2 / M R P 2$ gene than in carriers of other genotypes. For the remaining analyzed genes there was no significant difference in the incidence of progression or disease control depending on the presence of studied SNPs. Similarly, there was no significant difference in response to 
Table 1 Characteristics of patients and influence of clinical factors on response to second-line docetaxel therapy in NSCLC patients

\begin{tabular}{|c|c|c|c|c|c|c|c|c|}
\hline \multirow[t]{2}{*}{ Factors } & \multirow[t]{2}{*}{ Cases } & \multicolumn{4}{|c|}{ Response to treatment } & \multicolumn{3}{|c|}{ Overall survival } \\
\hline & & $\mathrm{SD}, \mathrm{PR}$ & $\mathrm{PD}$ & $p$ & $\begin{array}{l}\text { OR } \\
95 \% \mathrm{CI}\end{array}$ & $\begin{array}{l}\text { Median OS } \\
\text { (months) }\end{array}$ & $p$ & $\begin{array}{l}\mathrm{HR} \\
(95 \% \mathrm{CI})\end{array}$ \\
\hline Whole group & 58 & $14(24.1 \%)$ & $44(75.9 \%)$ & & & 4.25 & & \\
\hline \multicolumn{9}{|l|}{ Gender } \\
\hline $\begin{array}{l}\text { Male } \\
\text { Female }\end{array}$ & $\begin{array}{l}49(84.5 \%) \\
9(15.5 \%)\end{array}$ & $\begin{array}{l}11(22.4 \%) \\
3(33.3 \%)\end{array}$ & $\begin{array}{l}38(77.6 \%) \\
6(66.7 \%)\end{array}$ & 0.7813 & $\begin{array}{l}1.1633 \\
0.7146-1.8911\end{array}$ & $\begin{array}{l}4.5 \\
3.5\end{array}$ & 0.5061 & $\begin{array}{l}0.8234 \\
(0.4642-1.4603)\end{array}$ \\
\hline \multicolumn{9}{|l|}{ Age (years) } \\
\hline $\begin{array}{l}\text { Median ( } \pm \text { standard deviation }) \\
\leq 65 \\
>65\end{array}$ & $\begin{array}{l}61.7( \pm 7.7) \\
34(58.6 \%) \\
24(41.4 \%)\end{array}$ & $\begin{array}{l}8(23.5 \%) \\
6(25 \%)\end{array}$ & $\begin{array}{l}26(76.5 \%) \\
18(75 \%)\end{array}$ & 0.8551 & $\begin{array}{l}1.0196 \\
0.7577-1.372\end{array}$ & $\begin{array}{l}4.5 \\
6\end{array}$ & 0.6642 & $\begin{array}{l}1.2048 \\
(0.5194-2.7948)\end{array}$ \\
\hline \multicolumn{9}{|l|}{ Pathomorphological diagnosis } \\
\hline $\begin{array}{l}\text { Squamous cell carcinoma } \\
\text { Adenocarcinoma } \\
\text { Large cell carcinoma* } \\
\text { NOS (not-otherwise specified) NSCLC* }\end{array}$ & $\begin{array}{l}30(51.7 \%) \\
16(27.6 \%) \\
6(10.3 \%) \\
6(10.3 \%)\end{array}$ & $\begin{array}{l}10(33.3 \%) \\
1(6.3 \%)\end{array}$ & $\begin{array}{l}20(66.7 \%) \\
15(93.7 \%)\end{array}$ & 0.0677 & $\begin{array}{l}7.5 \\
0.8633-65.1592\end{array}$ & $\begin{array}{l}5 \\
4.25\end{array}$ & 0.7716 & $\begin{array}{l}1.1038 \\
(0.5666-2.1504)\end{array}$ \\
\hline \multicolumn{9}{|c|}{ Stage of disease } \\
\hline $\begin{array}{l}\text { IIIB } \\
\text { IV }\end{array}$ & $\begin{array}{l}19(32.8 \%) \\
39(67.2 \%)\end{array}$ & $\begin{array}{l}7(36.8 \%) \\
7(17.9 \%)\end{array}$ & $\begin{array}{l}12(63.2 \%) \\
32(82.1 \%)\end{array}$ & 0.2109 & $\begin{array}{l}0.7697 \\
0.5298-1.1183\end{array}$ & $\begin{array}{l}6.5 \\
3.5\end{array}$ & 0.1231 & $\begin{array}{l}0.6322 \\
(0.3529-1.1323)\end{array}$ \\
\hline \multicolumn{9}{|l|}{ Performance status (PS) } \\
\hline $\begin{array}{l}\mathrm{PS}>1 \\
\mathrm{PS} \leq 1\end{array}$ & $\begin{array}{l}23(39.7 \%) \\
35(60.3 \%)\end{array}$ & $\begin{array}{l}11(47.8 \%) \\
3(8.6 \%)\end{array}$ & $\begin{array}{l}12(52.2 \%) \\
32(91.4 \%)\end{array}$ & $\underline{0.0019}$ & $\underline{0.5707} \underline{0.3809-0.8549}$ & $\begin{array}{l}3 \\
8\end{array}$ & $\underline{0.0001}$ & $\begin{array}{l}3.4732 \\
(1.8949-6.3663)\end{array}$ \\
\hline \multicolumn{9}{|l|}{ Previous surgical treatment ${ }^{b}$} \\
\hline $\begin{array}{l}\text { Yes } \\
\text { No }\end{array}$ & $\begin{array}{l}5(8.6 \%) \\
53(91.4 \%)\end{array}$ & $\begin{array}{l}1(20 \%) \\
13(24.5 \%)\end{array}$ & $\begin{array}{l}4(80 \%) \\
40(75.5 \%)\end{array}$ & 0.7486 & $\begin{array}{l}1.06 \\
0.6662-1.6865\end{array}$ & & & \\
\hline \multicolumn{9}{|l|}{ Previous radiotherapy } \\
\hline $\begin{array}{l}\text { Yes } \\
\text { No }\end{array}$ & $\begin{array}{l}23(39.7 \%) \\
35(60.3 \%)\end{array}$ & $\begin{array}{l}7(30.4 \%) \\
7(71.9 \%)\end{array}$ & $\begin{array}{l}16(69.6 \%) \\
28(28.1 \%)\end{array}$ & 0.5520 & $\begin{array}{l}0.8696 \\
0.6333-1.194\end{array}$ & $\begin{array}{l}5 \\
3.5\end{array}$ & 0.5964 & $\begin{array}{l}0.8561 \\
(0.4817-1.5214)\end{array}$ \\
\hline \multicolumn{9}{|l|}{ Best response (first-line treatment) } \\
\hline $\begin{array}{l}\mathrm{PD} \\
\mathrm{CR}, \mathrm{PR}, \mathrm{SD}\end{array}$ & $\begin{array}{l}17(29.3 \%) \\
41(70.7 \%)\end{array}$ & $\begin{array}{l}2(11.8 \%) \\
12(29.3 \%)\end{array}$ & $\begin{array}{l}15(88.2 \%) \\
29(70.7 \%)\end{array}$ & 0.2797 & $\begin{array}{l}3.5753 \\
0.9595-1.6218\end{array}$ & $\begin{array}{l}2.5 \\
6\end{array}$ & 0.0793 & $\begin{array}{l}1.8728 \\
(0.9291-3.7748)\end{array}$ \\
\hline \multicolumn{9}{|c|}{ Duration of best response (to first-line treatment, months) } \\
\hline $\begin{array}{l}>12 \text { months } \\
\leq 12 \text { months }\end{array}$ & $\begin{array}{l}6(10.3 \%) \\
52(89.7 \%)\end{array}$ & $\begin{array}{l}3(50 \%) \\
11(21.2 \%)\end{array}$ & $\begin{array}{l}3(50 \%) \\
41(78.8 \%)\end{array}$ & 0.2893 & $\begin{array}{l}0.6341 \\
0.2814-1.429\end{array}$ & $\begin{array}{l}8.75 \\
3.5\end{array}$ & 0.1213 & $\begin{array}{l}0.5457 \\
(0.2536-1.1741)\end{array}$ \\
\hline \multicolumn{9}{|c|}{ Time from diagnosis to docetaxel therapy (months), Median ( \pm standard deviation) } \\
\hline $\begin{array}{l}>12 \\
\leq 12\end{array}$ & $\begin{array}{l}9( \pm 7.6) \\
22(37.9 \%) \\
36(62.1 \%)\end{array}$ & $\begin{array}{l}9(40.9 \%) \\
5(13.9 \%)\end{array}$ & $\begin{array}{l}13(59.1 \%) \\
31(86.1 \%)\end{array}$ & $\underline{0.0437}$ & $\underline{0.6862} \underline{0.4732-0.9951}$ & $\begin{array}{l}3 \\
7.5\end{array}$ & 0.1193 & $\begin{array}{l}1.5833 \\
(0.8881-2.8225)\end{array}$ \\
\hline \multicolumn{9}{|c|}{ Weight loss $(>5 \%)$ before docetaxel therapy } \\
\hline $\begin{array}{l}\text { Yes } \\
\text { No }\end{array}$ & $\begin{array}{l}18(31 \%) \\
40(69 \%)\end{array}$ & $\begin{array}{l}1(5.6 \%) \\
13(32.5 \%)\end{array}$ & $\begin{array}{l}17(94.4 \%) \\
27(67.5 \%)\end{array}$ & 0.0592 & $\begin{array}{l}1.3992 \\
1.0979-1.7832\end{array}$ & $\begin{array}{l}2.5 \\
6\end{array}$ & $\underline{0.0015}$ & $\begin{array}{l}3.5057 \\
(1.6132-7.6182)\end{array}$ \\
\hline \multicolumn{9}{|l|}{ Anemia before docetaxel therapy } \\
\hline $\begin{array}{l}\text { No } \\
\text { Yes }\end{array}$ & $\begin{array}{l}46(79.3 \%) \\
12(20.7 \%)\end{array}$ & $\begin{array}{l}5(41.7 \%) \\
9(19.6 \%)\end{array}$ & $\begin{array}{l}7(58.3 \%) \\
37(80.4 \%)\end{array}$ & 0.2245 & $\begin{array}{l}0.7252 \\
0.4403-1.1945\end{array}$ & $\begin{array}{l}4.5 \\
3.5\end{array}$ & 0.7062 & $\begin{array}{l}0.8786 \\
(0.4482-1.7222)\end{array}$ \\
\hline \multicolumn{9}{|l|}{ Toxicities of docetaxel therapy } \\
\hline $\begin{array}{l}\text { No } \\
\text { Yes }\end{array}$ & $\begin{array}{l}38(65.5 \%) \\
20(34.5 \%)\end{array}$ & $\begin{array}{l}5(25 \%) \\
9(23.7 \%)\end{array}$ & $\begin{array}{l}15(75 \%) \\
29(76.3 \%)\end{array}$ & 0.8325 & $\begin{array}{l}0.9828 \\
0.7216-1.3384\end{array}$ & $\begin{array}{l}4.5 \\
3\end{array}$ & 0.0791 & $\begin{array}{l}0.5532 \\
(0.2857-1.0712)\end{array}$ \\
\hline \multicolumn{9}{|c|}{ Subsequent systemic treatment after docetaxel termination ${ }^{\mathrm{a}}$} \\
\hline $\begin{array}{l}\text { No } \\
\text { Yes }\end{array}$ & $\begin{array}{l}40(69 \%) \\
18(31 \%)\end{array}$ & & & & & $\begin{array}{l}3 \\
7\end{array}$ & $\underline{0.0059}$ & $\begin{array}{l}2.2527 \\
(1.2639-4.0151)\end{array}$ \\
\hline
\end{tabular}

*not included in the statistical analysis

${ }^{a}$ including 13 patients treated with erlotinib without molecular selection

${ }^{\mathrm{b}}$ not included in the survival analysis (subgroup of patients, who underwent surgical treatment was not large enough) 
treatment depending on the level of expression of the TUBB3 gene. Relationships between clinical and molecular factors and occurrence of early progression are presented in Table 2 .

The median overall survival was 4.25 months ( \pm 9.6 months). In 24 patients (41.4\%) survival was longer than 6 months. Factors such as: good PS (HR $=0.26,95 \%$ CI: 0.12 $0.58, p<0.0001)$, disease control after docetaxel $(\mathrm{HR}=0.091$, 95\%CI: $0.014-0.63, p<0.0001$ ), long period from diagnosis to docetaxel (HR $=0.35,95 \% \mathrm{CI}: 0.17-0.71, p=0.0004)$, lack of significant weight loss prior to treatment $(\mathrm{HR}=0.48,95 \% \mathrm{CI}$ : $0.32-0.73, p=0.0021$ ) and the use of third-line treatment $(\mathrm{HR}=0.48,95 \% \mathrm{CI}: 0.24-0.94, p=0.0195)$ were associated with prolongation of survival beyond 6 months. Other clinical factors did not have a significant effect on the 6 months survival. We found no significant impact of individual SNPs or TUBB3 gene expression on the survival longer than 6 months.

Overall survival probability estimated by the Kaplan-Meier method was lower in patients with significant weight loss $(\mathrm{HR}=3.51,95 \mathrm{CI} \%: 1.61-7.62 ; p=0.0015)$, poor PS $(\mathrm{HR}=3.47,95 \mathrm{CI} \%: 1.89-6.37 ; p<0.0001)$, lack of disease control after docetaxel $(\mathrm{HR}=2.89,95 \mathrm{CI} \%: 1.60-5.21$; $p=0.0004$ ), and unable to receive third-line treatment (HR $=2.25,95 \mathrm{CI} \%: 1.26-4.01 ; p=0.0059)$. There was no significant impact of individual SNPs as well as TUBB3 gene expression on overall survival probability.

Although it seems that the applied treatment has a higher efficacy in the SCC subgroup of patients, we have failed to demonstrate a statistically significant effect on overall survival (Table 3). Kaplan-Meier survival curves of NSCLC and SCC patients with different $A B C C 2 / M R P 1$ genotypes were shown in Figs. 1 and 2.

According to the Cox model (general model fit: $p=0.0024)$ demographic, clinical and genetic factors that characterized the highest risk of survival shortening were: lack of third-line treatment (HR $=23.89,95 \%$ CI: $3.50-163.02$, $p=0.0013$ ), lack of best response to first-line therapy $(\mathrm{HR}=11.34,95 \% \mathrm{CI}: 2.04-63.11, p=0.0058)$, poor PS $(\mathrm{HR}=7.06,95 \% \mathrm{CI}: 1.59-31.32, p=0.0105)$, and CG or GG genotypes of $A B C C 2 / M R P 2$ gene $(\mathrm{HR}=5.21,95 \% \mathrm{CI}$ : $1.08-25.05, p=0.0404)$.

\section{Discussion}

TAX 317 [1] and TAX 320 [2] studies demonstrated effectiveness of docetaxel after failure of first-line chemotherapy. In the TAX 317 study, both the median survival ( 7.5 months) as well

Table 2 Influence of genetic alterations on response to second-line docetaxel therapy in NSCLC patients

\begin{tabular}{|c|c|c|c|c|c|c|c|c|}
\hline \multirow[t]{2}{*}{ Factors } & \multirow[t]{2}{*}{ Cases } & \multicolumn{4}{|c|}{ Response to treatment } & \multicolumn{3}{|c|}{ Overall survival } \\
\hline & & $\mathrm{SD}, \mathrm{PR}$ & PD & $p$ & $\begin{array}{l}\text { OR } \\
95 \% \mathrm{CI}\end{array}$ & $\begin{array}{l}\text { Median OS } \\
\text { (months) }\end{array}$ & $p$ & $\begin{array}{l}\mathrm{HR} \\
(95 \% \mathrm{CI})\end{array}$ \\
\hline \multicolumn{9}{|c|}{ MDR1 (c.3435 T > C) } \\
\hline $\mathrm{CC}$ & $8(15.4 \%)$ & $2(25 \%)$ & $6(75 \%)$ & \multirow[t]{3}{*}{0.7163} & \multirow[t]{3}{*}{-} & 7 & \multirow[t]{3}{*}{0.5541} & \multirow[t]{3}{*}{-} \\
\hline $\mathrm{CT}$ & $23(44.2 \%)$ & $3(13 \%)$ & $20(87 \%)$ & & & 3.5 & & \\
\hline TT & $21(40.4 \%)$ & $4(19 \%)$ & $17(81 \%)$ & & & 4.5 & & \\
\hline \multicolumn{9}{|c|}{ MDRl $($ c.3435 T > C) } \\
\hline $\mathrm{CC}$ & $8(15.4 \%)$ & $2(25 \%)$ & $6(75 \%)$ & \multirow[t]{2}{*}{0.5357} & 1.7619 & 7 & \multirow[t]{2}{*}{0.6007} & 0.7990 \\
\hline $\mathrm{CT}$ or TT & $44(84.6 \%)$ & $7(15.9 \%)$ & $37(84.1 \%)$ & & $0.2934-10.5811$ & 3.5 & & $0.3448-1.8516$ \\
\hline \multicolumn{9}{|c|}{ MDR1 $(\mathrm{c} .3435 \mathrm{~T}>\mathrm{C})$} \\
\hline TT & $21(40.4 \%)$ & $4(19.1 \%)$ & $17(80.9 \%)$ & \multirow[t]{2}{*}{0.7851} & 1.2235 & 3.75 & \multirow[t]{2}{*}{0.6050} & 0.8510 \\
\hline $\mathrm{CC}$ or $\mathrm{CT}$ & $31(59.6 \%)$ & $5(16.1 \%)$ & $26(83.9 \%)$ & & $0.287-5.216$ & 3.5 & & $0.4616-1.5686$ \\
\hline \multicolumn{9}{|c|}{$A B C C 2($ g. $99861014 C>\mathrm{G})$} \\
\hline $\mathrm{CC}$ & $11(21.1 \%)$ & $1(9.1 \%)$ & $10(90.9 \%)$ & \multirow[t]{3}{*}{0.0407} & - & 4.5 & \multirow[t]{3}{*}{0.6660} & \multirow[t]{3}{*}{-} \\
\hline $\mathrm{CG}$ & $20(38.5 \%)$ & $1(5 \%)$ & $19(95 \%)$ & & & 3 & & \\
\hline GG & $21(40.4 \%)$ & $7(33.3 \%)$ & $14(66.7 \%)$ & & & 4.5 & & \\
\hline \multicolumn{9}{|c|}{$A B C C 2($ g. $99861014 \mathrm{C}>\mathrm{G})$} \\
\hline $\mathrm{CC}$ & $11(21.1 \%)$ & $1(9.1 \%)$ & $10(90.9 \%)$ & \multirow[t]{2}{*}{0.4293} & 0.4125 & 3.5 & \multirow[t]{2}{*}{0.6211} & 1.2070 \\
\hline CG lub GG & $41(78.9 \%)$ & $8(19.5 \%)$ & $33(80.5 \%)$ & & $0.0459-3.7079$ & 4.5 & & $0.5724-2.5450$ \\
\hline \multicolumn{9}{|c|}{$A B C C 2$ (g.99861014C > G) } \\
\hline GG & $21(40.4 \%)$ & $7(33.3 \%)$ & $14(66.7 \%)$ & \multirow[t]{2}{*}{0.0221} & 7.2500 & 4.5 & \multirow[t]{2}{*}{0.3361} & 0.7426 \\
\hline $\mathrm{CC}$ or $\mathrm{CG}$ & $31(59.6 \%)$ & $2(6.5 \%)$ & $29(93.5 \%)$ & & $\overline{1.3297-39.5283}$ & 3.5 & & $0.4049-1.3619$ \\
\hline \multicolumn{9}{|c|}{ TUBB3 mRNA expression $(\triangle \mathrm{CT})$} \\
\hline Low & $22(42.3 \%)$ & $6(27.3 \%)$ & $16(72.7 \%)$ & \multirow[t]{2}{*}{0.1162} & 3.3750 & 8 & \multirow[t]{2}{*}{0.7539} & 0.8486 \\
\hline High & $30(57.7 \%)$ & $3(10 \%)$ & $27(90 \%)$ & & $0.7399-15.3946$ & 4 & & $0.3041-2.3682$ \\
\hline
\end{tabular}


Table 3 Influence of genetic alterations on response to second-line docetaxel therapy in subgroup of SCC patients

\begin{tabular}{|c|c|c|c|c|c|c|c|c|}
\hline \multirow[t]{2}{*}{ Factors } & \multirow[t]{2}{*}{ Cases } & \multicolumn{4}{|c|}{ Response to treatment } & \multicolumn{3}{|l|}{ Overall survival } \\
\hline & & $\mathrm{SD}, \mathrm{PR}$ & PD & $p$ & $\begin{array}{l}\text { OR } \\
95 \% \mathrm{CI}\end{array}$ & Median OS (months) & $p$ & $\begin{array}{l}\text { HR } \\
(95 \% \mathrm{CI})\end{array}$ \\
\hline \multicolumn{9}{|c|}{ MDR1 (c.3435 T > C) } \\
\hline $\mathrm{CC}$ & $6(20 \%)$ & $2(33.3 \%)$ & $4(66.7 \%)$ & \multirow[t]{3}{*}{0.5421} & \multirow[t]{3}{*}{-} & 8 & \multirow[t]{3}{*}{0.8798} & \multirow[t]{3}{*}{-} \\
\hline $\mathrm{CT}$ & $14(46.7 \%)$ & $2(14.3 \%)$ & $12(85.7 \%)$ & & & 2 & & \\
\hline TT & $10(33.3 \%)$ & $3(30 \%)$ & $7(70 \%)$ & & & 4.5 & & \\
\hline \multicolumn{9}{|c|}{ MDR1 $(\mathrm{c} .3435 \mathrm{~T}>\mathrm{C})$} \\
\hline $\mathrm{CC}$ & $6(20 \%)$ & $2(33.3 \%)$ & $4(66.7 \%)$ & \multirow[t]{2}{*}{0.5215} & 1.9 & 8 & \multirow[t]{2}{*}{0.6320} & 0.7224 \\
\hline CT or TT & $24(80 \%)$ & $5(20.8 \%)$ & $19(\%)$ & & $0.2669-13.5235$ & 4.5 & & $0.1909-2.7335$ \\
\hline \multicolumn{9}{|c|}{ MDRl $($ c.3435 T > C) } \\
\hline TT & $10(46.7 \%)$ & $3(30 \%)$ & $7(70 \%)$ & \multirow[t]{2}{*}{0.5439} & 1.7143 & 4.5 & \multirow[t]{2}{*}{0.9511} & 0.9736 \\
\hline $\mathrm{CC}$ or $\mathrm{CT}$ & $20(53.3 \%)$ & $4(20 \%)$ & $16(80 \%)$ & & $0.3007-9.7731$ & 4.5 & & $0.4138-2.2905$ \\
\hline \multicolumn{9}{|c|}{$A B C C 2($ g. $99861014 C>\mathrm{G})$} \\
\hline $\mathrm{CC}$ & $7(23.3 \%)$ & $1(14.3 \%)$ & $6(85.7 \%)$ & \multirow[t]{3}{*}{$\underline{0.0224}$} & - & 3 & \multirow[t]{3}{*}{0.3521} & \multirow[t]{3}{*}{-} \\
\hline CG & $14(46.7 \%)$ & $1(7.1 \%)$ & $13(92.9 \%)$ & & & 3 & & \\
\hline GG & $9(30 \%)$ & $5(55.6 \%)$ & $4(44.4 \%)$ & & & 8.5 & & \\
\hline \multicolumn{9}{|c|}{$A B C C 2($ g.99861014C > G) } \\
\hline $\mathrm{CC}$ & $7(23.3 \%)$ & $1(14.3 \%)$ & $6(85.7 \%)$ & \multirow[t]{2}{*}{0.5248} & 0.4722 & 3 & \multirow[t]{2}{*}{0.6527} & 1.2863 \\
\hline CG lub GG & $23(76.7 \%)$ & $6(26.1 \%)$ & $17(73.9 \%)$ & & $0.0468-4.7697$ & 4.5 & & $0.4296-3.8518$ \\
\hline \multicolumn{9}{|c|}{$A B C C 2$ (g.99861014C > G) } \\
\hline GG & $9(30 \%)$ & $5(55.6 \%)$ & $4(44.4 \%)$ & \multirow[t]{2}{*}{$\underline{0.0135}$} & 11.875 & 8.5 & \multirow[t]{2}{*}{0.1227} & 0.5051 \\
\hline $\mathrm{CC}$ or $\mathrm{CG}$ & $21(70 \%)$ & $2(9.5 \%)$ & $19(90.5 \%)$ & & $\overline{1.6684}-84.5221$ & 3 & & $0.2122-1.2024$ \\
\hline \multicolumn{9}{|c|}{ TUBB3 mRNA expression $(\triangle \mathrm{CT})$} \\
\hline Low & $14(46.7 \%)$ & $5(35.7 \%)$ & $9(64.3 \%)$ & \multirow[t]{2}{*}{0.1483} & 3.8889 & 8 & \multirow[t]{2}{*}{0.6164} & 0.7114 \\
\hline High & $16(53.3 \%)$ & $2(12.5 \%)$ & $14(87.5 \%)$ & & $0.6168-24.5182$ & 4 & & $0.1878-2.6956$ \\
\hline
\end{tabular}

as the 12 month survival rate (37\%) were significantly higher in patients treated with docetaxel compared to patients receiving best supportive care (4.6 months and $11 \%$, retrospectively). In the TAX 320 study, response occurred in $7.5-11.9 \%$ of patients depending on the dose of docetaxel, and in $1 \%$ of patients in the control group receiving vinorelbine or ifosfamide. 12 month survival rate was $32 \%$ in patients treated with docetaxel and 19\% in the control group. Second line treatment can be used in patients with PS scored at $0-1$, and in exceptional cases with PS $=2$. Patients with PS $>2$ should not be treated [3]. The results obtained in our study confirm that PS is critical, affecting all of the analyzed parameters of treatment effectiveness: the risk of early progression, overall survival, and risk of death. The median survival of patients in good PS (PS $\leq 1)$ was 8 months, while patients in worse condition (PS > 1) only survived 3 months.

One of the few studies which address the problem of determining predictors of benefit from second-line treatment in NSCLC is a phase III study by Hanna et al. [4] comparing the efficacy of docetaxel and pemetrexed. Analysis showed significantly longer survival among women, patients with stage IIIB, in good PS (PS $\leq 1)$ and with objective response to firstline treatment. Unfortunately, the study involved patients who qualified under strict criteria $-89 \%$ of them were in good or very good PS (PS $\leq 1)$. Median survival for patients with PS $=0$ was 12.7 months, compared to 8.3 months for patients with $\mathrm{PS}=1$ and 2.6 months for $\mathrm{PS}=2$. Median survival was 9.4 months for women, and 7.2 months for men. Similar differences were observed in patients differentiated by stage of disease (IIIB vs. IV). Patients with objective response to firstline treatment achieved longer survival than patients with stable disease (median 15.8 vs 10.5 months), and patients from both above groups fared better than those who progressed (median survival of 4.6 months).

In the univariate analysis, Weiss et al. [5] found a longer median survival in patients with non-squamous NSCLC and with first-line response $>3$ months. In authors study PS of $60.3 \%$ of patients was rated as 2 , which resulted in shorter median survival. Multivariate analysis showed that in addition to the factors mentioned in the analysis by Weiss et al., survival was also affected by substantial weight loss $(>5 \%)$, male sex, lack of surgical treatment prior to chemotherapy and third-line treatment after docetaxel.

Tibaldi et al. [6] treated older patients ( $70-83$ years, median 74 years) with reduced dose $\left(37.5 \mathrm{mg} / \mathrm{m}^{2}\right)$ of docetaxel. Despite the reduction $21 \%$ of patients reached partial remission, and another $36 \%$ stable disease. Those results were better than in both TAX studies. This probably isn't associated 
Fig. 1 Survival curves of NSCLC patients with different ABCC2/MRP1 genotypes

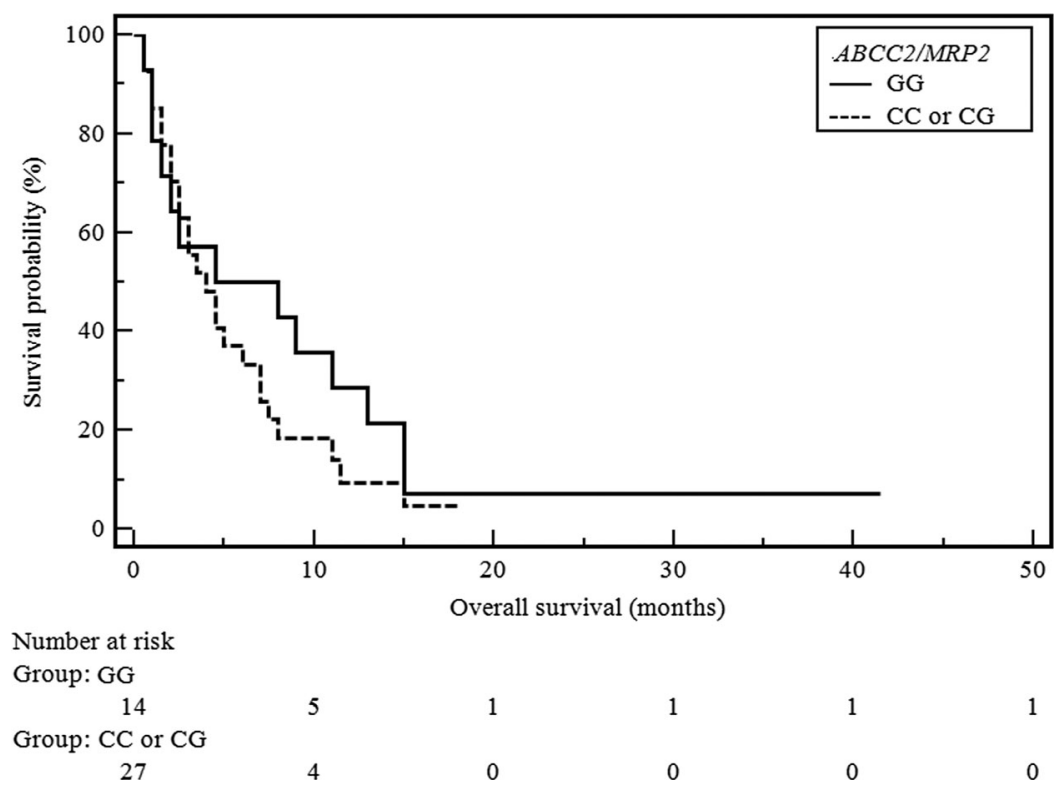

with greater effectiveness of docetaxel in this age group, but with slower course of the disease in the elderly.

In a study evaluating the effect of prior first-line treatment on effectiveness of second-line docetaxel in patients with NSCLC, Macedo-Pérez et al. [7] showed that long ( $>6$ months) response to first-line treatment is associated with significant increase in time to progression (TTP) and overall survival.

Our results confirmed crucial predictive role of such factors as: poor PS, significant weight loss and short time from diagnosis to docetaxel treatment. However, we have found no effect of gender, age, stage of disease or pathological diagnosis on overall survival of NSCLC patients treated with docetaxel. Interpretation of impact of the third-line treatment after docetaxel is difficult. We found that third-line therapy can significantly increase the probability of survival longer than 6 months. However, such treatment is used in patients with good PS, which is the most important predictive factor.

In the absence of independent predictors that could help qualify for second-line docetaxel treatment, currently the biggest hope rests on molecular research. It has been shown that patients with high expression of class III $\beta$-tubulin benefit less from paclitaxel treatment [8]. In meta-analysis of 10 clinical trials in which NSCLC was treated with paclitaxel and/or vinorelbine, Zhang et al. [9] demonstrated a correlation between low expression of mRNA for the TUBB3 gene and favorable outcomes - in the group of patients with low expression percentage of objective response was higher, and median survival longer. Kaira et al. [10] evaluated effects of class III
Fig. 2 Survival curves of SCC patients with different $A B C C 2 /$ MRP1 genotypes

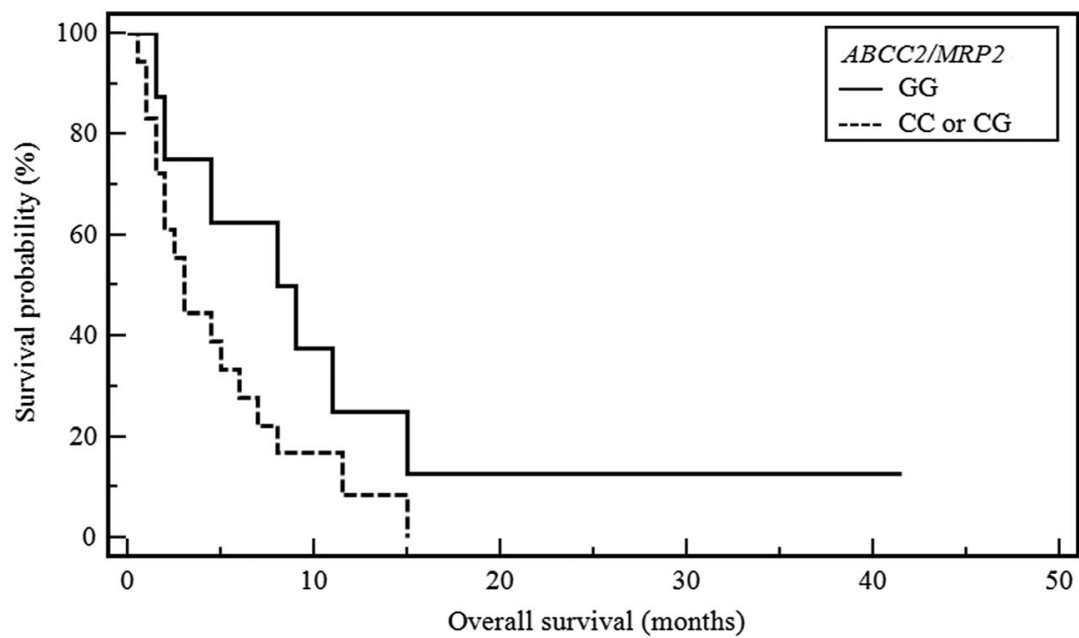

Number at risk

Group: GG

8

18
3

2
1

0
1

0
1

0 
$\beta$-tubulin expression on progression-free survival and overall survival in patients with NSCLC treated with taxanes. Researchers demonstrated that in patients treated with docetaxel high $T U B B 3$ expression is predictive for short time to progression and concluded that it is associated with resistance to taxanes. In our study no significant difference in response to therapy, probability of survival longer than 6 months, or overall survival was found depending on the level of mRNA expression of the $T U B B 3$ gene.

In normal lung, ABCB1 is expressed on the surface of bronchi and at the plasma membrane of alveolar macrophages. In lung cancer, ABCB1 expression is initially low, but this may change after exposure to chemotherapy as part of acquired drug resistance. ABCB1 confers resistance to cytotoxic drugs, including etoposide and cisplatin. ABCC2 is expressed at low levels in normal lung tissue and at high levels in lung cancer. It has higher expression in poorly differentiated compared to well-differentiated tumors. This suggests that these tumors may quickly develop resistance to anti-cancer agents. In tumor cell lines, ABCC2 mRNA overexpression was associated with resistance to etoposide, vincristine, cisplatin, doxorubicin and epirubicin.

Haenish $\mathrm{S}$ et al. [11] showed that $A B C B 1 M D R 1$ and $A B C C 2 / M R P 2$ SNPs modulate the expression of multidrug resistance protein in the unaffected renal cortex of renal cell carcinoma patients. In Meyer et al. [12] study, high MRP2 expression was correlated with $A B C C 2$ gene variants (especially rs717620) in liver tissue. Such correlation was not found between $A B C B 1 / M D R 1$ SNPs and MDR1 expression. Allelic variants of the $A B C C 2 / M R P 2$ gene have also been associated with toxicity induced by chemotherapy agents and lung cancer survival. Few reports suggest that polymorphisms in $A B C B 1 /$ $M D R 1$ significantly influence the therapeutic response in lung cancer, although these findings are not always concordant. Therefore, $M D R$ genes polymorphisms possibly contribute to inter-individual differences in drug and xenobiotics elimination and chemotherapy efficacy. However, the role of $M D R$ genes SNPs in docetaxel susceptibility or resistance needs further investigation in non-small cell lung cancer patients.

Previous studies related to polymorphisms of $M D R$ genes and chemotherapy effectiveness were conducted mainly in the populations of Asian origin. Isla et al. [13] studied the effects of SNPs of ERCC1, XPD, RRM1, ABCB1/MDR1 genes, and mRNA expression for ERCC1 on overall survival in patients with advanced NSCLC treated with docetaxel and cisplatin. Authors ascertained that evaluation of ERCC1 gene SNPs may be an important factor for effectiveness of platinumbased chemotherapy. However, analysis of the MDRl gene polymorphisms showed no statistically significant results.

In their 2008 paper, Kiyotani et al. [14] studied the effects of SNPs in seven genes potentially associated with transport and metabolism of docetaxel (CYP3A4, CYP3A5, ABCB1/ MDR1, ABCC2/MRP2, SLCO1B3, NR1I2 and NR1I3) in
Japanese patients with NSCLC. Polymorphisms in $A B C C 2$ (rs12762549) and SLCO1B3 (rs11045585) genes were significantly associated with the risk of docetaxel induced leucopenia. In combined evaluation of both polymorphisms, patients with unfavorable genotype proved to have significantly higher risk of neutropenia compared to patients with favorable genotype. Assessment system based on the genotyping of patients allowed to correctly classify $69.2 \%$ of patients with severe neutropenia and $75.7 \%$ of patients without this adverse effect to the appropriate categories, confirming that the SNPs in $A B C C 2$ and $S L C O 1 B 3$ genes may be a predictive factor for docetaxel induced neutropenia.

In a paper describing Caucasian patients, Campa et al. [15] characterized the genetic variability of genes $A B C B 1 / M D R 1$, $A B C C 2$ and $A B C G 2$ in lung cancer patients treated with chemotherapy. Of the studied polymorphisms, SNP rs717620 of the $A B C C 2$ gene has been linked to differences in treatment response, progression-free survival and overall survival of patients with small cell lung cancer (SCLC), but this effect was not observed in NSCLC. Lewis et al. [16] in their study which included 64 American NSCLC patients receiving docetaxel showed that SNP rs12762549 of the $A B C C 2$ gene is associated with reduced clearance of the docetaxel. In our study, in the model including demographic, clinical and genetic factors, SNP rs12762549 of the $A B C C 2$ gene $(\mathrm{C} / \mathrm{G}$ and $\mathrm{G} / \mathrm{G}$ genotypes) was one of the factors that had a significant impact on the risk of shortened overall survival.

\section{Conclusions}

Based on the results of our study it can be concluded that second-line docetaxel monotherapy in the treatment of NSCLC has limited efficacy, and qualification of patients for this type of treatment requires a careful analysis of available prognostic and predictive factors. Factors assessed in this study mainly have prognostic and not predictive value, which is consistent with the results of other researchers. Molecular studies, especially of the genes whose protein products are responsible for the clearance of cytostatics from tumor cells, may be useful in the selection of patients for docetaxel monotherapy. Finding reliable molecular predictors for this type of therapy however requires randomized, prospective clinical trials.

\section{Compliance with Ethical Standards}

Conflict of Interest The authors declare that they have no conflict of interest.

Ethical Approval All procedures performed in studies involving human participants were in accordance with the ethical standards of the institutional and/or national research committee and with the 1964 Helsinki declaration and its later amendments or comparable ethical standards. 
Informed Consent Informed consent was obtained from all individual participants included in the study.

Open Access This article is distributed under the terms of the Creative Commons Attribution 4.0 International License (http:// creativecommons.org/licenses/by/4.0/), which permits unrestricted use, distribution, and reproduction in any medium, provided you give appropriate credit to the original author(s) and the source, provide a link to the Creative Commons license, and indicate if changes were made.

\section{References}

1. Shepherd FA, Dancey J, Ramlau R, Mattson K, Gralla R, O'Rourke M et al (2000) Prospective randomized trial of docetaxel versus best supportive care in patients with non-small-cell lung cancer previously treated with patinum-based chemotherapy. J Clin Oncol 18: 2095-2103

2. Fossella FV, De Vore R, Kerr RN, Crawford J, Natale RR, Dunphy F et al (2000) Randomized phase III trial of docetaxel versus vinorelbine or ifosfamide in patients with advanced non-small-cell lung cancer previously treated with platinum-containing chemotherapy regimens. The TAX 320 non-small cell lung cancer study group. J Clin Oncol 18(12):2354-2362

3. Stinchcombe TE, Sociński MA (2008) Consideration for second line therapy of non-small cell lung cancer. Oncologist 13(supl 1): 28-36

4. Hanna N, Shepherd FA, Fossella FV, Pereira JR, De Marinis F, von Pawel J et al (2004) Randomized phase III trial of pemetrexed versus docetaxel in patients with non-small-cell lung cancer previously treated with chemotherapy. J Clin Oncol 1:1589-1597

5. Weiss GJ, Rosell R, Fossella F, Perry M, Stahel R, Barata F et al (2007) The impact of induction chemotherapy on the outcome of second-line therapy with pemetrexed or docetaxel in patients with advanced non-small-cell lung cancer. Ann Oncol 18(3):453-460

6. Tibaldi C, Bernardini I, Chella A, Russo F, Vasile E, Malventi M et al (2006) Second-line chemotherapy with a modified schedule of docetaxel in elderly patients with advanced-stage non-small-cell lung cancer. Clin Lung Cancer 7(6):401-405

7. Macedo-Pérez EO, Morales-Oyarvide V, Mendoza-García VO, Dorantes-Gallareta Y, Flores-Estrada D, Arrieta O (2014) Long progression-free survival with first-line paclitaxel plus platinum is associated with improved response and progression-free survival with second-line docetaxel in advanced non-small-cell lung cancer. Cancer Chemother Pharmacol 74(4):681-690

8. Sève P, Dumontet CI (2008) Class III beta-tubulin a predictive factor in patients receiving tubulin-binding agents? Lancet Oncol 9(2):168-175

9. Zhang HL, Ruan L, Zheng LM, Whyte D, Tzeng CM, Zhou XW (2012) Association between class III $\beta$-tubulin expression and response to paclitaxel/vinorebine-based chemotherapy for non-small cell lung cancer: a meta-analysis. Lung Cancer 77(1):9-15

10. Kaira K, Takahashi T, Murakami H, Shukuya T, Kenmotsu H, Ono A et al (2013) The role of $\beta$ III-tubulin in non-small cell lung cancer patients treated by taxane-based chemotherapy. Int J Clin Oncol 18(3):371-379

11. Haenisch S, Zimmermann U, Dazert E, Wruck CJ, Dazert P, Siegmund W, Kroemer HK, Warzok RW, Cascorbi I (2007) Influence of polymorphisms of $\mathrm{ABCB} 1$ and $\mathrm{ABCC} 2$ on mRNA and protein expression in normal and cancerous kidney cortex. Pharmacogenomics J 7(1):56-65

12. Meier Y, Pauli-Magnus C, Zanger UM, Klein K, Schaeffeler E, Nussler AK, Nussler N, Eichelbaum M, Meier PJ, Stieger B (2006) Interindividual variability of canalicular ATP-binding cassette $(\mathrm{ABC})$-transporter expression in human liver. Hepatology 44: $62-74$

13. Isla D, Sarries C, Rosell R, Alonso G, Domine M, Taron M et al (2004) Single nucleotide polymorphisms and outcome in docetaxel-cisplatin-treated advanced non-small-cell lung cancer. Ann Oncol 15(8):1194-1203

14. Kiyotani K, Mushiroda T, Kubo M, Zembutsu H, Sugiyama Y, Nakamura Y (2008) Association of genetic polymorphisms in SLCO1B3 and ABCC2 with docetaxel-induced leukopenia. Cancer Sci 99(5):967-972

15. Campa D, Muller P, Edler L, Knoefel L, Barale R, Heussel CP, Thomas M, Canzian F, Risch AA (2012) Comprehensive study of polymorphisms in $\mathrm{ABCB} 1, \mathrm{ABCC} 2$ and $\mathrm{ABCG} 2$ and lung cancer chemotherapy response and prognosis. Int J Cancer 131:2920 2928

16. Lewis LD, Miller AA, Owzar K, Bies RR, Markova S, Jiang C et al (2013) The relationship of polymorphisms in ABCC2 and SLCO1B3 with docetaxel pharmacokinetics and neutropenia: CALGB 60805. Pharmacogenet Genomics 23(1):29-33 